\title{
3D Bioprinting of Hybrid Materials for Regenerative Medicine: Implementation in Innovative Small and Medium-Sized Enterprises (SMEs)
}

\author{
ROXANA MIOARA PITICESCU, ${ }^{1}$ LAURA MADALINA CURSARU ${ }^{10},{ }^{1,4}$ \\ DAN NASTASE CIOBOTA, ${ }^{1}$ SINZIANA ISTRATE, ${ }^{2}$ \\ and DUMITRU ULIERU ${ }^{3}$
}

\begin{abstract}
1.-National Research-Development Institute for Non-Ferrous and Rare Metals (INCDMNRIMNR), 102 Biruintei Blvd, 077145 Pantelimon, Ilfov, Romania. 2.-Ophthalmology Department, "Carol Davila" University of Medicine and Pharmacy, 050474 Bucharest, Romania. 3.-S.C. SITEX 45 S.R.L, Blvd. Ghica Tei, Bl. 40, Ap. 2, Dept. 2114, 023709 Bucharest, Romania. 4.-e-mail: mpopescu@imnr.ro
\end{abstract}

Three-dimensional printing has significant potential as a fabrication method for synthetic scaffolds for regenerative medicine. The advantages of fabricating scaffolds using 3D printing are numerous, including the ability to create complex geometries and porosities that mimic the anatomical structures of organs and tissues. Three-dimensional printing can be used to create orbital implants, which replace the bony cavity surrounding the eye after eye removal. In the present article, an original 3D printing method is developed for the production of three-dimensional structures based on hydroxyapatite and polyurethane diol, which can be further used for the fabrication of orbital implants with interconnected porosity. For this purpose, hybrid nanostructured powders were prepared by hydrothermal synthesis and further used as raw materials for $3 \mathrm{D}$ printing of porous structures. Innovative porous $3 \mathrm{D}$ structures were tested in vitro and in vivo. The test results showed the vascularization capacity of the 3D implant and remodeling of the tissue.

\section{INTRODUCTION}

A person with one eye missing, whatever the cause, may suffer psychologically as well as physically. ${ }^{1}$ In most cases an orbital implant is used to restore orbital volume (the bone cavity where the eyeball is located). An orbital implant maintains the natural appearance of the orbit and is a support for the artificial eye (ocular prosthesis) when surgical removal of the eyeball is needed. The most common causes leading to the removal of the eye are ocular traumas or eyeball infections but also congenital diseases of the eyeball or even cancer. ${ }^{2,3}$

The first implant was developed about 100 years ago, and attempts to make one have included the most unusual materials: glass, gold, silver, wool, rubber, cartilage, bone, fat, magnets, silicon, cork, titanium, acrylate, asbestos, cellulose, paraffin, sponge, polymethylmethacrylate (PMMA), polyethylene (PE) and hydroxyapatite (HAp). Orbital implants may be alloplastic or autoplastic, porous (including natural or synthetic hydroxyapatite) or non-porous (silicon). ${ }^{4}$ The first generation of orbital implants could not give the artificial eye natural movement. The appearance and natural movement of the artificial eye were obtained using a natural material: ocean coral, whose porous structure is similar to human bone. ${ }^{5}$

In 1983, Dr. Arthur Perry made the first porous orbital implant from a marine coral whose structure was modified from calcium carbonate to calcium phosphate (mainly hydroxyapatite) by a hydrothermal reaction that simultaneously removes proteins and residual materials. ${ }^{6}$ Its biologically inert structure and porosity resembling the Haversian human bone system allow it to be accepted by the body without the risk of rejection encountered in other materials. On the other hand, the porous architecture allows fibrovascular growth within the orbital implant. As the implant vascularizes through this 
fibroblast growth, it becomes embedded in the orbit, thus lowering the risk of migration, a risk commonly encountered in other types of implants. ${ }^{7}$ The degree of vascularization used to perform a second step by drilling and implanting a screw is evaluated by CT (computed tomography), performed 6 months postoperatively. ${ }^{8}$

The implant design has progressed significantly in recent years with the use of porous devices that, theoretically, have few complications and improve the cosmetic appearance. However, these benefits come after a long period of healing, sometimes with unsatisfactory results, complications and prolonged periods of hospitalization. Also, the patient's reintegration into society takes longer. Many have to wait for the implant's vascularity, and then they are subjected to other new surgical procedures for the second drilling step.

Although the first artificial eye was introduced 100 years ago, the best material for the "perfect artificial eye" remains to be discovered. The implant models, such as the Bio-Eye (USA), FCI3 (France), Medpore (USA) and Molteno M-sphere (USA) Bioceramic Orbital Implant (FCI Ophtalmic), have progressed significantly in recent years because of the use of porous materials, with the theoretical advantages of fewer complications and improved aesthetics. ${ }^{9}$ In adults, these implants will be subjected to a high rate of expulsion if they are not wrapped in polyglactin mesh, autologous sclera or fascia lata due to the material roughness. For this reason, finding a material to wrap the useful implant and attach extraocular muscles that will allow a natural movement of the prosthesis is a challenge. Children's implants are all the more important as maintaining the volume of the orbital cavity is essential for the symmetrical development of the orbit and for preventing its decrease during the growth process. The tendency for dermal graft implants for orbital reconstruction in children is a $25 \%$ regression of the dermal implant and central ulceration within the first 6 months of implantation requiring repeated reinterventions.

In 2010, FCI, France, and Innovia, USA, proposed an extensible Occlusal Tissue Expander implant for children to prevent microphthalmia and anophthalmia, but there are still reports regarding the need for a surgical postimplantation review. Designing the architecture of scaffold structures on the macro, micro and nano levels is important for the structural conditions, nutritional transport and cellular matrix. Macroarchitecture is the general form of the device and can be complex (e.g., patient specificity, anatomical features). Microarchitecture reflects the tissue architecture (e.g., pore size, shape, porosity, spatial distribution and pore interconnection). Nanoarchitecture involves surface modification (e.g., attachment of biomolecules for cell adhesion, proliferation, and differentiation). Although an ideal scaffold material will take all these factors into account, there are still some challenges related to the selection of biomaterials and the specificity of $3 \mathrm{D}$ forms. ${ }^{10}$

The main goal of the present article is to highlight the advantages of an original $3 \mathrm{D}$ printing method developed in this study for the production of threedimensional structures based on hydroxyapatite and polyurethane diol, which can be further used for the fabrication of orbital implants with interconnected porosity.

For the first time, hybrid nanostructured powders based on hydroxyapatite and polyurethane diol, with homogeneous chemical structure and controlled morphology, were prepared by hydrothermal synthesis in high-pressure conditions and further used as raw materials for $3 \mathrm{D}$ printing of porous structures (scaffolds) by mixing with water soluble binders and extrusion agents.

\section{MATERIALS AND METHODS}

\section{Hydrothermal Synthesis of Hydroxyapatite- Polyurethane-Based Hybrid Materials Fol- lowed by Spray Drying}

Hybrid materials were synthesized starting from $\mathrm{Ca}$ and $\mathrm{P}$ soluble salts as hydroxyapatite precursors: $\mathrm{Ca}\left(\mathrm{NO}_{3}\right)_{2} * 4 \mathrm{H}_{2} \mathrm{O}$ and $\mathrm{NH}_{4} \mathrm{H}_{2} \mathrm{PO}_{4}$, respectively. $\mathrm{A}$ commercial polyurethane diol solution was added, and the solution $\mathrm{pH}$ was adjusted to 10 , resulting in a hydrid precursor suspension that was further transferred to the HP Systems high-pressure autoclave (France) and subjected to hydrothermal treatment in isostatic pressure conditions (1000 bar) and temperature $<120^{\circ} \mathrm{C}$ for $3 \mathrm{~h}$. After hydrothermal treatment, the nanostructured hybrid powder was washed with distilled water until neutral $\mathrm{pH}$ was reached and further spray-dried using a LabPLANT dryer. $^{11}$

\section{Characterization of Hybrid HAp-PU Powders}

Prior to scaffold fabrication starting from hydrothermally synthesized powders, as-obtained hybrids were characterized from structural, morphologic and biocompatible points of view.

A Bruker D8 Advance x-ray diffractometer operated at $40 \mathrm{kV}$ and $40 \mathrm{~mA}$, using $\mathrm{CuK} \alpha$ radiation with a wavelength of $1.5405 \mathrm{~A}$ in Bragg's diffraction angle ' $2 \theta$ ' ranging from $6^{\circ}$ to $81^{\circ}$, was used to study the structural properties. The size and morphology of the prepared hybrid nanopowders were obtained using a Tecnai G2 F30 S-TWIN, FEI transmission electron microscope (TEM). For preliminary in vitro experiments, hybrid powders based on hydroxyapatite and polyurethane were pressed at 1-3 tons using a manual hydraulic press (Specac). Small 11mm-diameter disks were obtained and used for cell viability measurements. Hap-PU disks were sterilized and then tested through contact with human endothelial progenitor cells (huEPC). Biocompatible 
properties were studied using the XTT Cell Proliferation Assay.

\section{Preparation of Filler Paste for 3D Bioprinting}

HAp-PU nanostructured hybrid powders synthesized in high-pressure hydrothermal conditions (1000 bar), as presented above, were mechanically mixed with a carefully selected water-soluble polymer binder and an extrusion bioprinting solution (crosslinking agent) to obtain HAp-PU-based pastes used as a feedstock in the 3D Bioprinting technique. $^{11,12}$

\section{Fabrication of 3D Scaffolds Based on Hydrox- yapatite-Polyurethane Hybrids}

The 3D structures were obtained from HAP-PUbased pastes prepared as described above using the 3D Bioprinting technique and BioScaffolder (SYSENG, Germany) device, connected via USB port to a computer on which the Bioscaffolder SW 3.0 software is installed. This software allows the configuration of the $3 \mathrm{D}$ object to be created in this program or imported from a CAD-CAM program as well as the setting of the printing process parameters for the 3D object (scaffold), made by depositing several layers of extruded fibers.

\section{Setting Dispersion Parameters}

The deposition parameters are set according to the data presented in Table I. Then, the hybrid nanostructured paste is inserted into the BioScaffolder syringe. Once the deposition substrate has been fixed, the machine calibrated by setting the origin (the starting point of $3 \mathrm{D}$ deposition) on the three axes $(x, y, z)$ and the 3D CAD data files, STL type (standard 3D format in Rapid Prototyping), imported, the actual deposition begins.

\section{Optical Microscopy (OM) Analysis of 3D Structures}

For this purpose, an optical microscope with polarized light, reflected and transmitted (Axio Imager A1 m, Carl Zeiss Microimaging Gmbh), equipped with a digital camera for image acquisition and AxioVision Release 4.8.1 dedicated software for image processing, was used.

\section{Morphologic Characterization of 3D Structures Using Scanning Electron Microscopy (SEM)}

Morphologic characterization was carried out by scanning electron microscopy (SEM) using a Quanta Inspect F50 FEG microscope (resolution $1.4 \mathrm{~nm}$ ) equipped with an energy dispersive x-ray detector (EDAX), (FEI Co., Eindhoven, The Netherlands). Particle size measurements were performed with DigitalMicrograph $^{\mathrm{TM}}$ (Gatan Inc., Pleasanton, CA, USA) image acquisition and processing software.

\section{In Vivo Test of HAp-PU 3D Scaffolds}

The anatomopathologic blocks were immersed in $10 \%$ formalin, embedded in paraffin and then cut using a Thermo Fisher Microm EC $1150 \mathrm{H}$ and Leica EG1150H. The sections were prepared for immunohistochemistry staining using primary antibodies for fibroblasts and vascular tissue.

\section{RESULTS AND DISCUSSIONS}

\section{Hydrothermal Synthesis of Hydroxyapatite- Polyurethane-Based Hybrid Materials in High-Pressure Conditions}

The hydrothermal method is a well-known and attractive technique for producing pure nanocrystalline, highly homogeneous nanoparticles in a single step, in aqueous medium, with low energy consumption. ${ }^{13-16}$ The hydrothermal method has been proven to be an effective, convenient and environmentally friendly process.

Hydrothermal synthesis at high pressure is characterized by the following main advantages: (1) low energy consumption, developed by applying pressure (the energy consumed to increase the temperature by 5 units is equal to that required to increase the pressure by 4000 units in the system); (2) the negative $\Delta V$ value between the total molar volume of reaction products and the total molar volume of reactants (shifting equilibrium to compounds with the smallest volume); (3) improvement of the

Table I. Deposition parameters

\begin{tabular}{lc}
\hline Component/parameter & Value \\
\hline Polyethylene syringe (ml) & 10 \\
Nozzle diameter (mm) & $0.4-0.8$ \\
Nozzle length (mm) & 31 \\
3D object & Cube, $l=20$ mm, octagon or cylinder \\
The movement speed of the deposition head (mm/min) & $400-700$ \\
The rotation speed of the spindle motor drive piston extruder (rot/s) & $0.05-0.06$ \\
Model (the angle of rotation between two successive layers) & $45^{\circ}$ and $90^{\circ}$ \\
Layer thickness (mm) & $80 \%$ of nozzle diameter \\
The distance between extruded fibers (mm) & $1.3-1.6$ \\
\hline
\end{tabular}


chemical reactivity; (4) single-step synthesis of nanocrystalline materials without the need for further heat treatment; (5) retaining the unaltered structure of the polymer because of the low reaction temperature $\left(<120^{\circ} \mathrm{C}\right)$; (6) environment-friendly technology and closed autoclave, without the release of toxic compounds.

\section{Characterization of Hybrid HAp-PU Powders}

\section{X-Ray Diffraction (XRD) Patterns}

$\mathrm{X}$-ray diffraction confirms the presence of hydroxyapatite as the major crystalline phase in both cases: sample A with $80 \%$ hydroxyapatite and sample B with 50\% hydroxyapatite. The mean crystallites determined with the Scherrer equation are $12-12.5 \mathrm{~nm}$. Figure 1 illustrates the $\mathrm{x}$-ray diffractograms of samples A and B.

\section{TEM Analysis}

An example of TEM analysis for hydrothermally synthesized HAp-PU samples at 1000 bar is shown in Fig. 2.

TEM images of different nanopowders, namely sample A (with 80\% HAp) and sample C (20\% HAp), are shown in Fig. 2. It can be observed that both types of samples are made of spheric particles (Fig. 2a and d). Figure 2c reveals the typical morphology of HAp crystallites prepared by the hydrothermal method-whiskers. In sample $\mathrm{C}$, one can observe the presence of HAp whiskers embedded in the polymer matrix (Fig. 2e).

\section{Biocompatible Properties (Preliminary In Vitro Test)}

Hap-PU disks prepared for preliminary in vitro experiments are based on hybrid powders with $80 \%$ HAp and denoted as P1-P10. A cell suspension containing $1.2 \times 10^{6}$ cell $/ \mathrm{ml}$ was prepared so that each volume of $50 \mu \mathrm{l}$ contained 60,000 cells. Hap-PU sterile disks were placed in the ultra-low attachment (ULA) 24-well plate and kept in culture medium $\mathrm{EGM}_{2}$ at $37^{\circ} \mathrm{C}, 5 \% \mathrm{CO}_{2}$ for $1 \mathrm{~h}$. After this time, the medium in which the disks were incubated was removed, and $50 \mu \mathrm{l}$ of cell suspension was added to the disks. A control well (containing only cells) was also added. After an additional hour in the incubator, $400 \mu \mathrm{l} \mathrm{EGM}_{2} /$ well was added. The results showed that there were no cell agglomerates around the disk. The P1, P2 and P5 disks are biocompatible and can support viable human endothelial progenitor cells (huEPC) compared with those cultivated directly on the ULA (Fig. 3).

\section{Evaluation of Cell Viability by XTT Assay}

After 4 days from seeding, cell viability was tested by the XTT method for wells containing HAp-PU disks (denoted as Px disc) and wells containing cells after disk removal (denoted as Px cells).
The results showed increased viability for P1 (155\%), P2 (147\%) and P5 (135\%) disks, associated with an increased number of cells: $26.900,23.775$ and 18.750 versus 3.625 cells in the control (Fig. 4). However, it should be taken into account that the control cells were grown differently from the normal cultivation conditions for huEPCs.

\section{Fabrication of Personalized 3D Structures}

The reconstruction parameters are based on the assumption that different medical imaging devices will give the same response on the Hounsfield scale for the same areas of interest, but the fine-tuning step is needed to cover the variations that may occur in that range. In the last step, different smoothing algorithms with specific parameters can be tried to optimize the external surface quality, in terms of accuracy and reduction of edge effects, given by the weathered representation of a curved surface. For the fabrication stage, the new eye-implant model can be used in machines that can manufacture the porosity-controlled structures by additive manufacturing techniques. Add-on fabrication (AM) technology allows the manufacture of very complex lattice structures that cannot be obtained through conventional technologies. Porous structures have similar or superior qualities to the corresponding compact parts. Thus, they usually have larger internal surfaces and increased ratios between strength and weight. Another important advantage is that an interconnected pore structure facilitates the penetration of the fluid and the biologic cells, finally resulting in a gradual increase of the tissue once they have been fixed to the structure.

Lattice microstructures offer huge potential for use in designing cell structures with small specific weights. Experiments have shown that the mechanical response of lattice structures is governed by the microarchitecture of the structure. Research in the field has concerned the production and testing of such light structures with their possible applications. Attention has focused on the relationship between microstructure-mechanical properties-deformation, a relationship that allows optimization of the structure design according to the mechanical loads to which it is subjected. The ultimate goal is to obtain personalized implants that possess mechanical features similar to those of the prosthetic organ.

Three-dimensional printing is a highly versatile technique to fabricate complex structures with high precision and nanometer resolution. The principle of this technique is based on the extrusion of continuous filaments (inks) in a layer-by-layer sequence using computer-aided design (CAD) tools. This method is capable of fabricating structures with feature sizes on the microscale $(0.1-100 \mu \mathrm{m})$ and mesoscale $(>100 \mu \mathrm{m})$, depending on the nozzle diameter. The ability to fabricate a variety of structures requires good control over the formulation and rheologic behavior of the inks and printing 


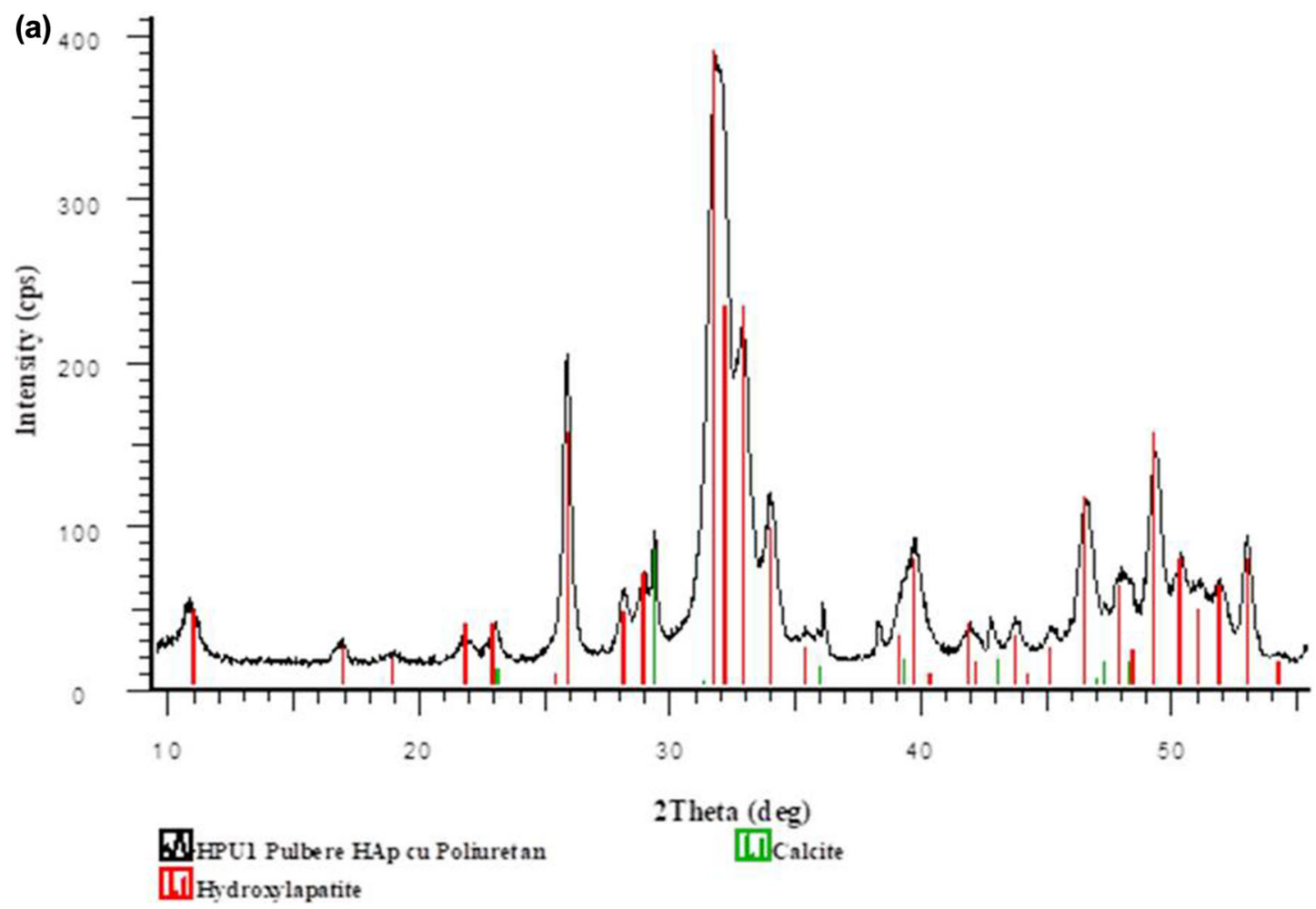

(b)

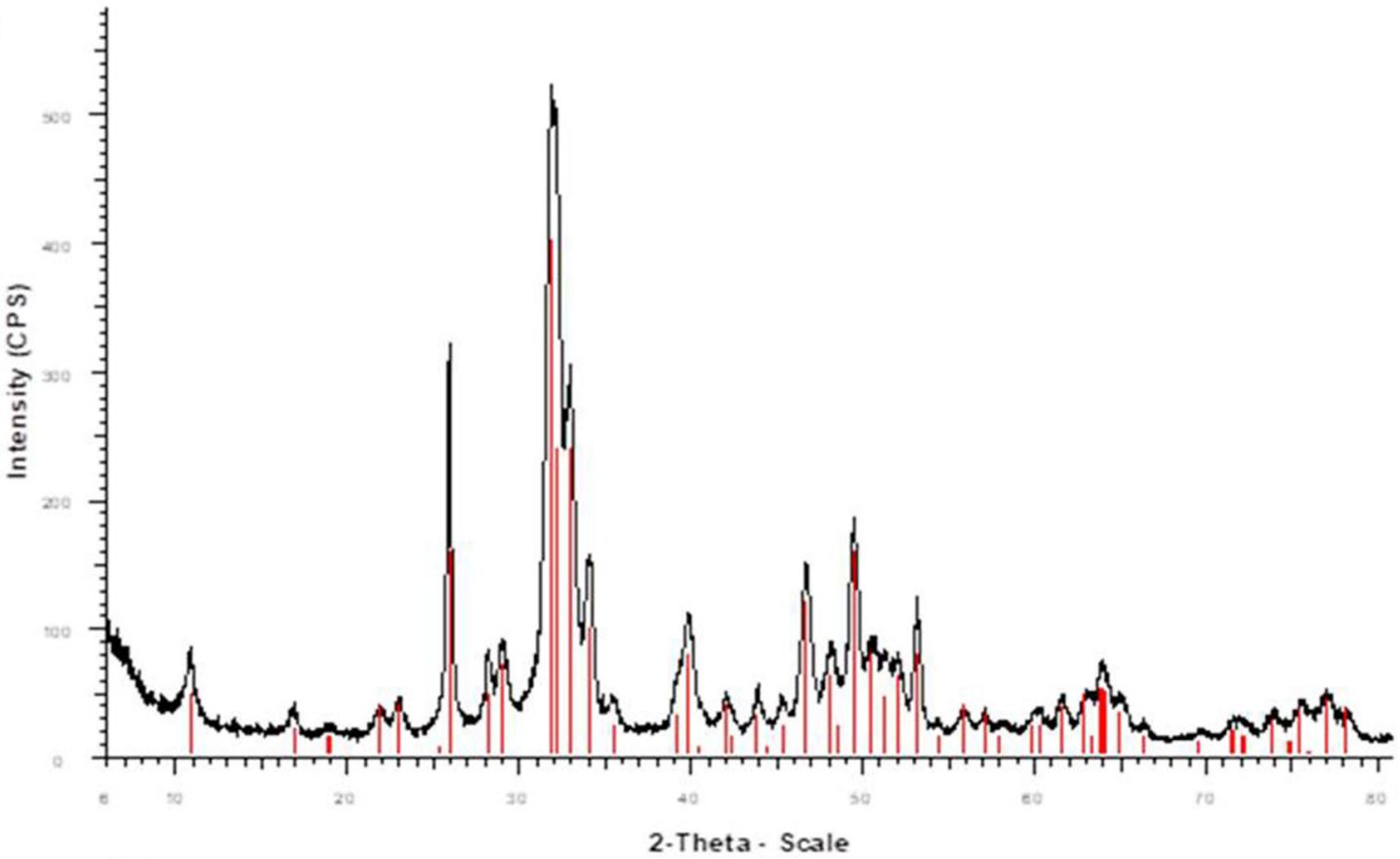

圆E: 234-1 HPUS

I.1. Hydroxyla patto

Fig. 1. X-ray diffractograms of (a) sample A ( $80 \%$ HAp); (b) sample B (50\% HAp).

parameters (velocity printing, pressure extrusion). ${ }^{17}$ The fundamental requirement for any lattice structure is that it is self-supporting (to bear on itself) without the need for inside supports. Another key aspect for the use of porous structures is pore interconnectivity. Porosity is defined as the total percentage of bare spaces in a solid and is a morphologic property independent of the material.

Figure 5 presents an example of $3 \mathrm{D}$ scaffolds based on HAp-PU powder. 

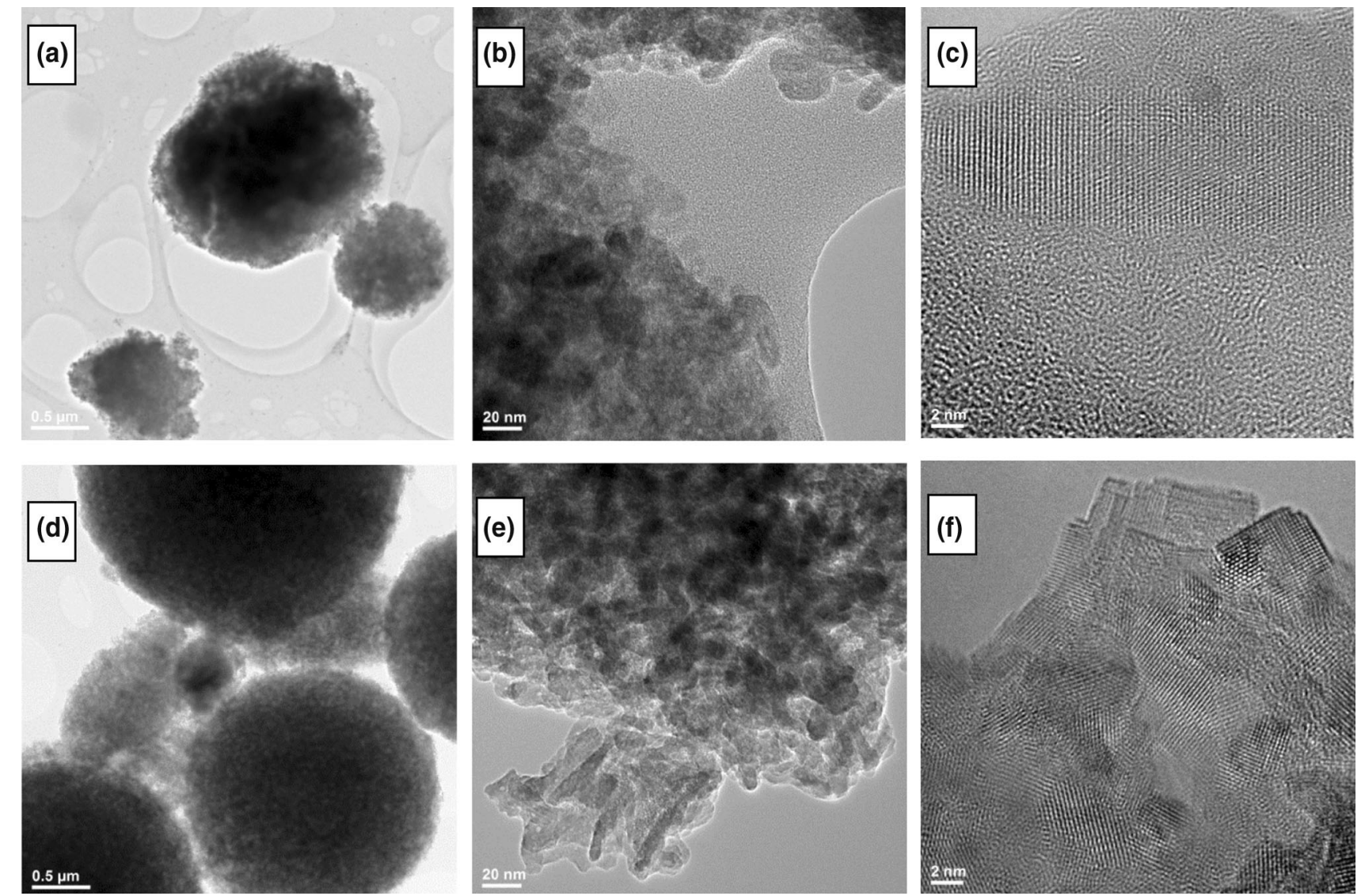

Fig. 2. TEM images of sample $A(80 \% \mathrm{HAp})(a, b, c)$; sample $C(20 \% \mathrm{HAp})(\mathrm{d}, \mathrm{e}, \mathrm{f})$

\section{Optical Microscopy Characterization of 3D Structures}

Three-dimensional scaffolds based on HAp-PU hybrid powder were analyzed by optical microscopy. In the example presented in Fig. 6, the diameter of an extruded fiber is about $992.5 \mu \mathrm{m}$, and the distance between two extruded fibers is around $471.2 \mu \mathrm{m}$.

\section{Morphologic Characterization of 3D Structures Using Scanning Electron Microscopy (SEM)}

An example of SEM analysis for HAp-PU-based 3D hybrid structures is shown in Fig. 7. The SEM images in Fig. 7 show the morphology of 3D structures based on hydroxyapatite-polyurethane-diol nanostructured hybrid powders. BioScaffolder fibers can be seen at $90^{\circ}$ and $45^{\circ}$ angles. Their thickness $(800 \mu \mathrm{m}-1 \mathrm{~mm})$ is in accordance with optical microscopy results, where the sample presented in Fig. 6 is $992.5 \mu \mathrm{m}$ in diameter. The distance between two extruded fibers varies between 663.23-837.29 $\mu \mathrm{m}$ (Fig. 8a) and 451.74-618.77 $\mu \mathrm{m}$ (Fig. 8d). These values depend on the setting parameters of the Bioscaffolder SW 3.0 software but could also be correlated with the particular structure and composition of the extruded fibers. Unlike other composite materials used for 3D printing of an orbital implant, in the present article the polymeric component has been present in the fiber structure since the raw powder preparation. Figure $7 \mathrm{~b}$ and e shows that both samples of the investigated 3D structures are composed of spherical particles with diameters of 2-4 microns and 3-6 microns, respectively, as a result of the spraydrying process. The EDX spectrum confirms the presence of the $\mathrm{Ca}, \mathrm{P}$ and $\mathrm{O}$ elements in the hydroxyapatite structure but also of the $\mathrm{C}$ in the polyurethane structure.

\section{In Vivo Test of HAp-PU 3D Scaffolds}

Figure 8a illustrates the HAp-PU 3D implant 3 months after the surgical intervention with new fibrovascular tissue grown inside the 3D structure (CD31-positive staining of a endothelial cell; black arrow). The osseous tissue is present in the 3D structure with osteoclasts (white arrow) that can degrade and remodel the HAp-PU implant as a positive marker for osteointegration.

Figure $8 \mathrm{~b}$ presents the HAP-PU 3D implant 3 months after surgical intervention with fibroblasts (white arrow) indicating scaffold formation (normal vascular endothelium is stained in dark 

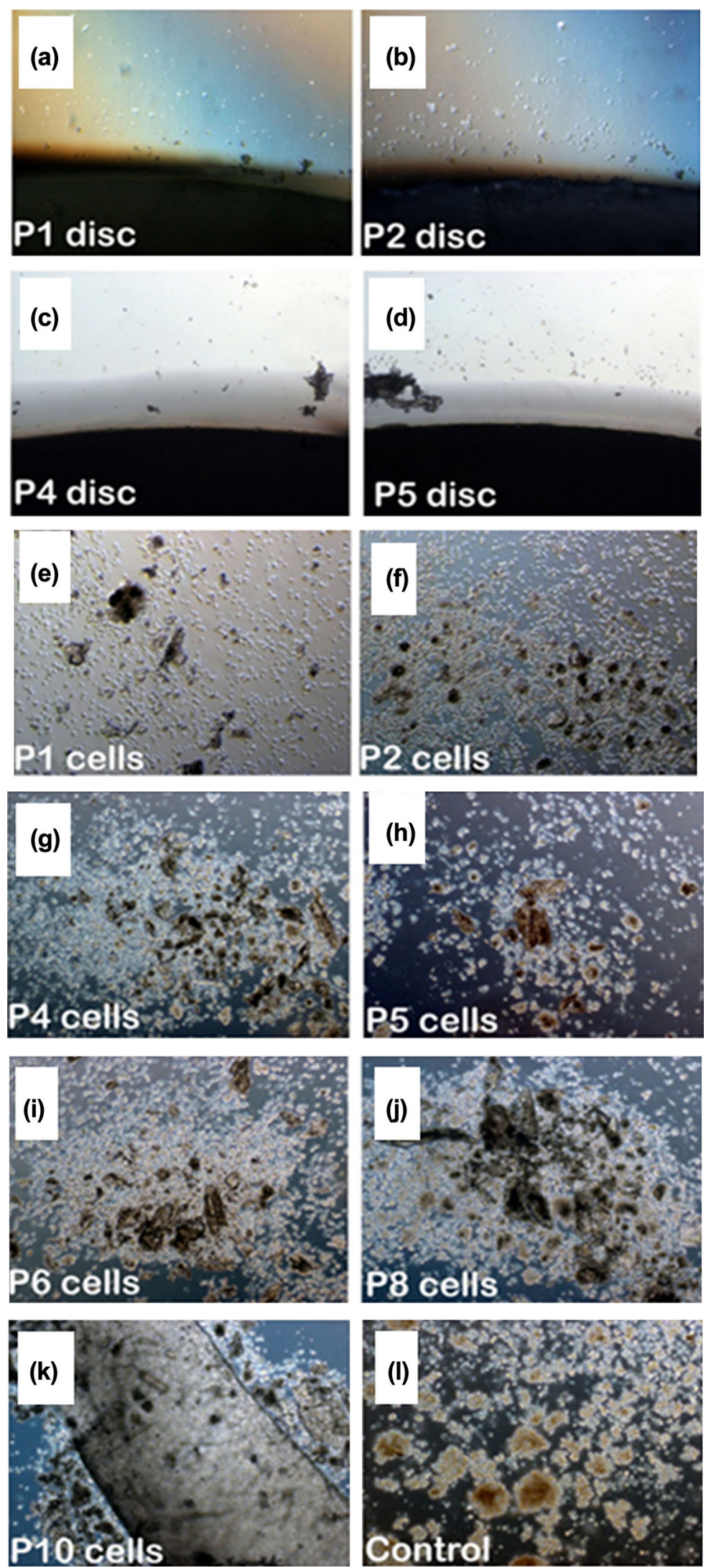

Fig. 3. Optical microscopy image illustrating HAp-PU disks (a-d) and cells left in the well (e-k) after removing the disks, $72 \mathrm{~h}$ from seeding; (I) cells left in the control well. 

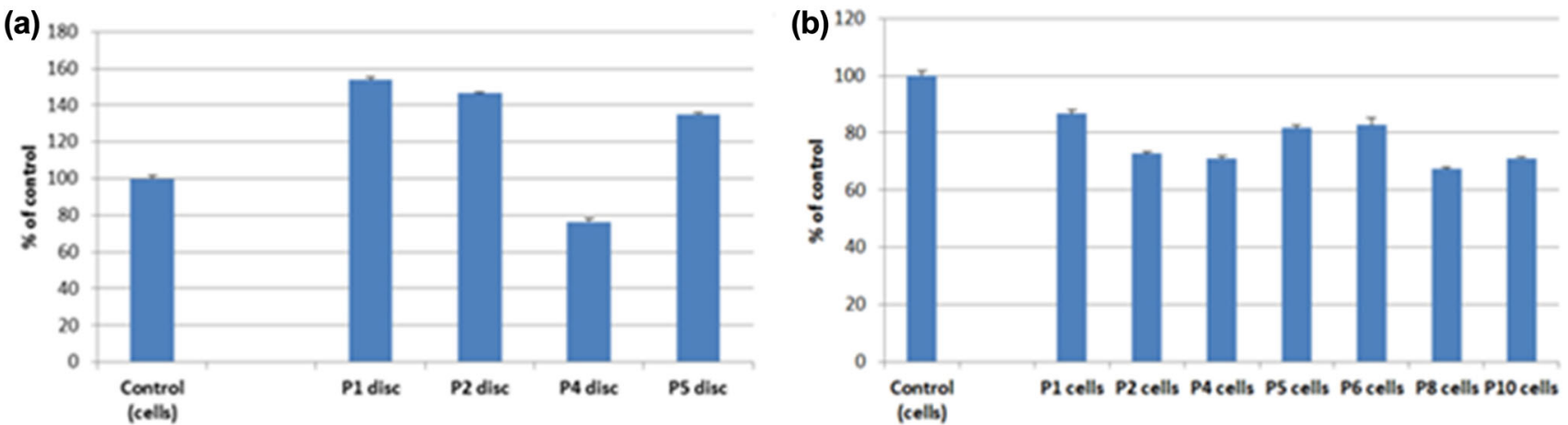

Fig. 4. Viability of huEPCs grown on disks (a) and viability of the cells remaining in the wells after disk removal (b).
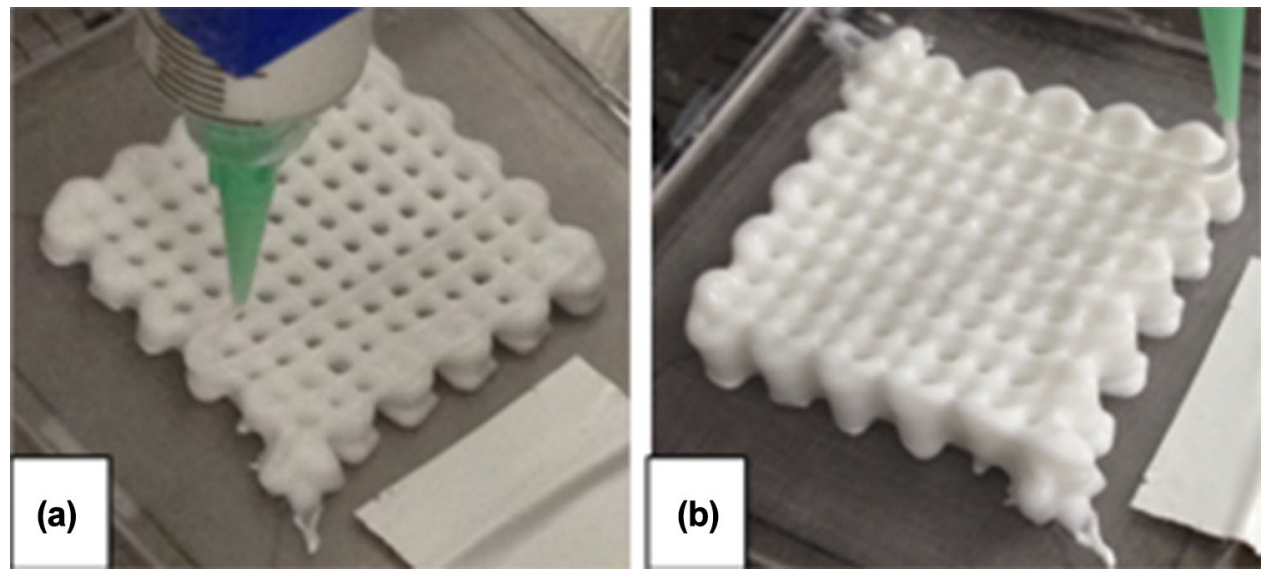

Fig. 5. Three-dimensional scaffolds obtained by 3D printing using Bioscaffolder (SYSENG): (a) during deposition; (b) after deposition.

brown; white arrowhead). The adjacent connective tissue of the sclera is negative for CD34 staining.

The best methodology for in vivo evaluation of 3Dengineered acellular scaffolds was considered CD3, CD 31, CD 34 and CD 163 immunohistochemistry staining as a control method for biocompatibility, tissue degradation, inflammation and recellularization. ${ }^{18}$ Satisfactory vascularization is a prerequisite for the survival of grafts and the integration of new tissue with existing tissue. ${ }^{19}$ Thus, we demonstrate the capacity of the surrounding vascular tissue to migrate in the 3D printed implant with new vessel formation and also remodeling of the tissue demonstrated by the presence of osteoclasts in the 3D implant.

\section{Implementation in Innovative Small and Medium-Sized Enterprises (SMEs)-Orig- inality of the Proposed Technology}

The fabrication technology of 3D structures presented above, with potential applications as orbital implants, is easy to translate to SMEs because of the low energy consumption, environmentally friendly process, inexpensive products and existing market niche at the national and European levels. Market analysis of orbital implants is briefly presented below.

\section{Emerging Market}

Eye cancer is a general term used to describe many types of tumors that occur in various parts of the eye. Melanoma is the most common type of primary intraocular cancer in adults. Primary eye cancers can occur at any age, but the risk increases as people get older. Retinoblastoma is the most common type of eye cancer in children. Ocular malignancies account for $3-4 \%$ of all malignancies in children and include retinoblastoma (incidence 44.2 and 67.9 per million births ${ }^{6}$ ) and rhabdomyosarcoma sarcoma (incidence of 4.5 per million). There are about 12 million cancer survivors in the USA; many were diagnosed when they were $<21$ years. Over the last 30 years, improved treatment strategies and better supportive care have resulted in increased survival rates for many childhood cancers. Today, $75 \%$ of children and adolescents with cancer will survive $\geq 10$ years after treatment.

The American Cancer Society's estimate for eye cancer in the USA for 2013 is 2800 new cases of cancer (primarily melanoma) of the eye and orbit: 1490 in males and 1310 in females. In both the US and Europe, eye cancer (melanoma in most cases) incidence in the elderly is about $5-7.5$ cases per million people per year; for people $>50$ years old, the incidence rate increases to around 21 per million 

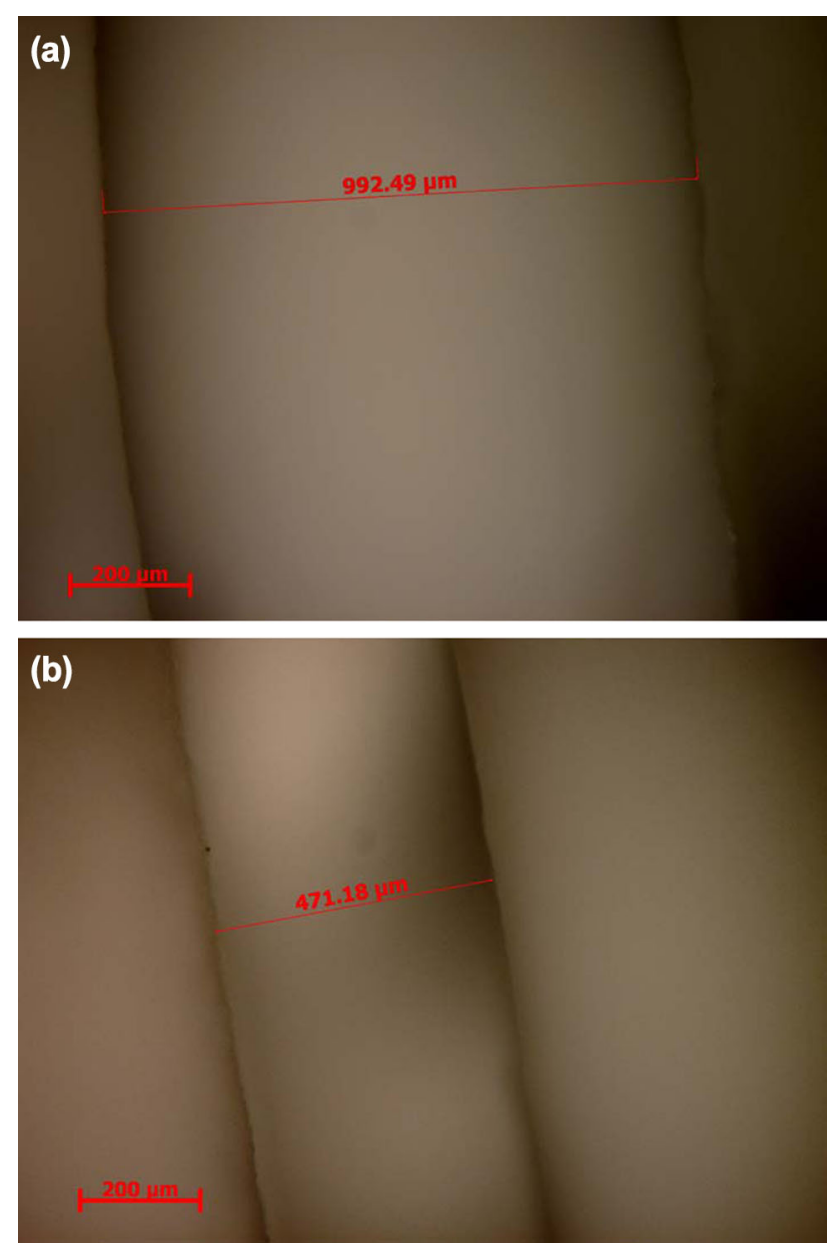

Fig. 6. Optical micrographs of a 3D printed sample showing: (a) the diameter of an extruded fiber; (b) the distance between two extruded fibers per year. Approximately $50 \%$ of patients with ocular melanoma will develop metastases by $10-15$ years after diagnosis (a small percentage of people will develop metastases even later, i.e., 20-25 years after their initial diagnosis). Metastatic disease is universally fatal. This $50 \%$ mortality rate has remained unchanged despite advances in treating the primary eye tumors. More research is urgently needed to improve patient outcomes. When an eye is removed, an orbital implant is used to replace the volume in the orbit that was occupied by the eye.

Today's most commonly used orbital implants are solid (non-porous) polymeric spheres (silicone, PMMA), Allen-type implants, the AlphaSphere, bovine HA (porous), coralline HA (porous), synthetic HA (porous), porous PE, porous alumina and Guthoff implants. ${ }^{3}$ These implants present some drawbacks such as high costs, risk of conjunctival abrasion, poor mechanical strength and fibrovascular ingrowth.

In our case, the proposed 3D printing technology is easy for SMEs to implement because it is not based on commercial powders but on powders that can be produced on demand and command. Our 3D printing technique is an emerging technology and could reach the maturity level in 5 years. The implant fabricated using Bioscaffolder equipment will have internal, interconnected pores, a higher vascularization rate and control of possible residual metastasis. It is expected that the ingrowth process of the orbital tissue into the implant to will take place in $<6-9$ months (the actual process time). Inorganic (Hap)-organic (polyurethane) nanostructured hybrid materials combining the properties of
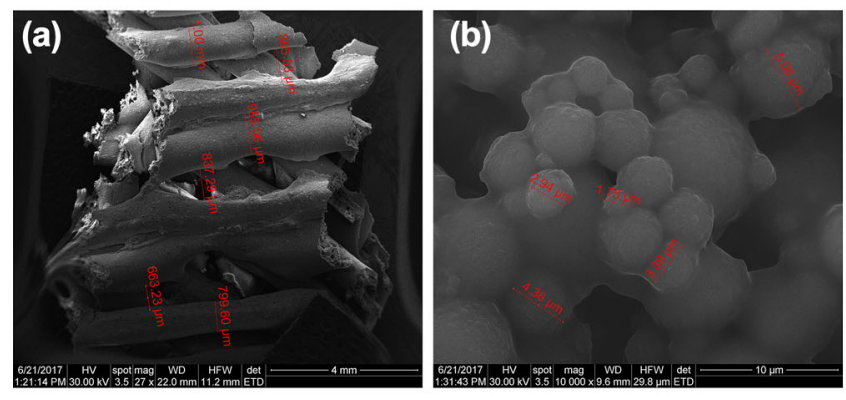

(c)
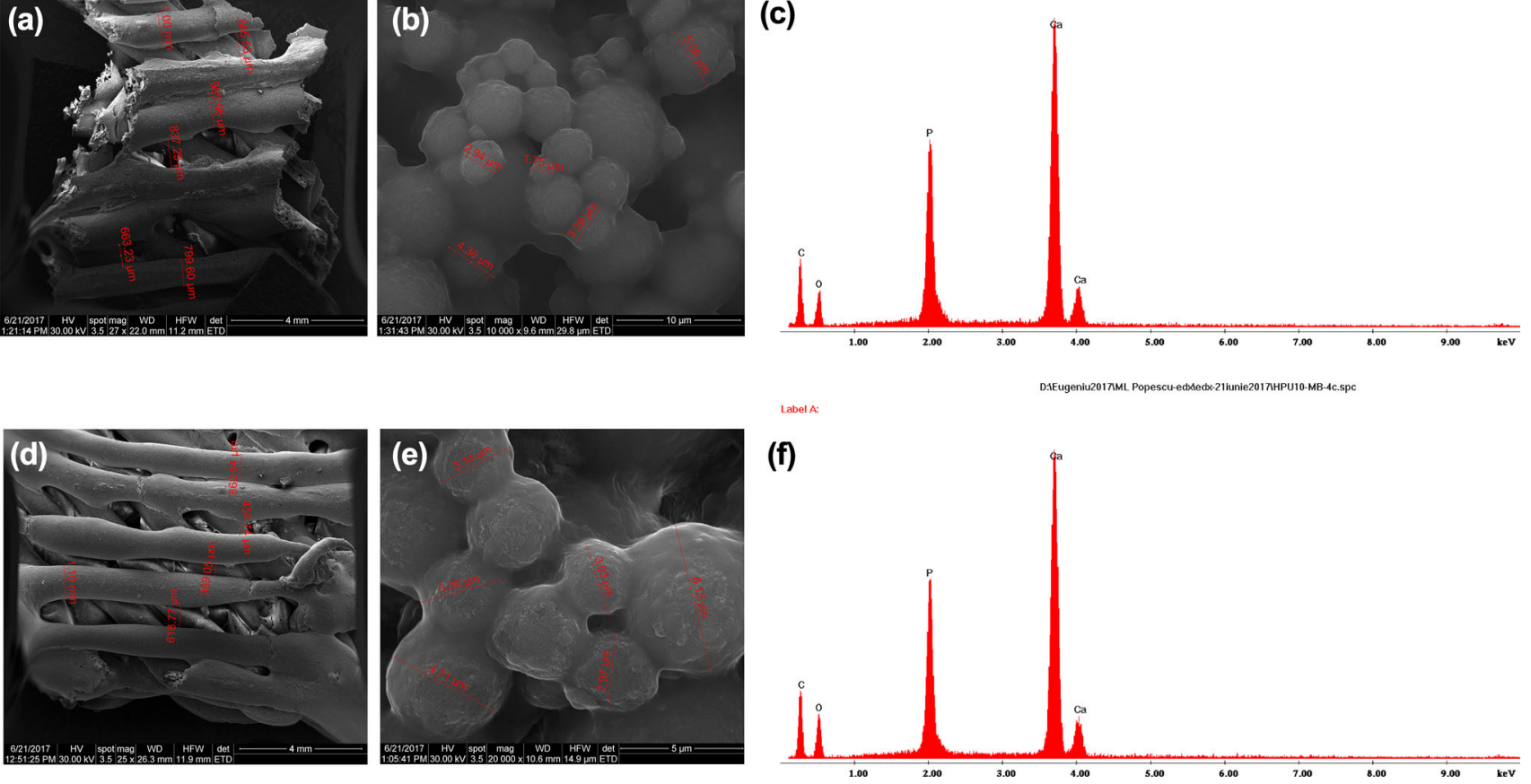

Fig. 7. SEM images of 3D printed samples at various scales (a, b, d, e); EDX spectra of samples (c, f). 

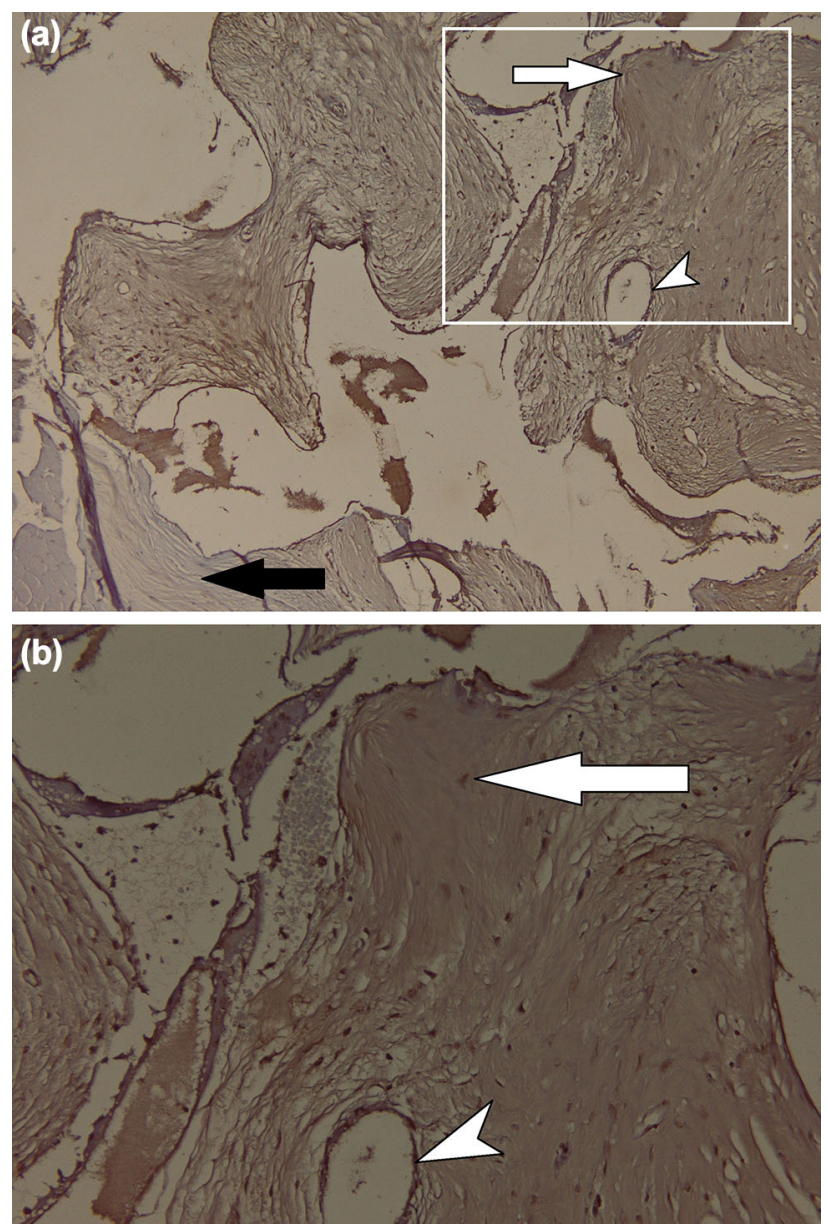

Fig. 8. (a) Immunohistochemistry CD31 + staining, $\times 100$; (b) immunohistochemistry CD $34+$ staining, $\times 200$.

each component are used as an alternative to current practice, which employs orbital implants made of non-porous silicone, HAp or porous PE.

Geographic distribution of the eye implant market: From a geographic point of view, the eye implant market is segmented into several geographic regions: North America, Latin America, Europe, Asia-Pacific, excluding Japan, the Middle East and Africa. North America and Europe will be key areas for the eye implant market. In the AsiaPacific region, a positive increase is expected because of the demographic structure of the population and the development of the health infrastructure in the region.

Active companies for the global eye implant market: The market leaders in the global eye implant market include Novartis AG, Pfizer Inc., Staar Surgical, Johnson \& Johnson Services, Inc., Bausch \& Lomb Inc., Morcher ${ }^{\circledR} \mathrm{GmbH}$ and others.

The growth factors of the eye implant market include population aging and positive growth due to technologic advances in implant making, such as the technology of multifocal intraocular lenses. At the same time, the demands of patients, particularly young ones, will increase regarding their appearance and physiognomy requirements. Factors for decreasing market demand include the reduction of staff for ophthalmic surgery and also the side effects resulting from the application of ocular implants [Ocular Implants Market to Witness Double Digit CAGR by 2024, developed and published by iCrowdNewswire, Aug 15, 2017$].$

\section{Commercial Details Regarding Orbital Implant Products and Manufacturers in Europe and the USA}

One of the leading manufacturers in Europe is FCI SAS Paris, which makes the orbital implants from hydroxyapatite HAp: a synthetic orbital implant having porosity of 200-500 microns [Ocular Implants Market Competitive Dynamics USA \& Global Outlook 2024]. Another noteworthy manufacturer in Europe is Network Medical Products, Ltd., Coronet House, UK.

\section{CONCLUSION}

The orbital implant presented in this article is a novelty at both the national and global levels. Achieving this is a medical challenge because there are currently no commercially available orbital implants made of nanostructured hybrid hydroxyapatite-polyurethane-based materials.

For the first time, hybrid organic-inorganic materials based on hydroxyapatite and commercial water-soluble polyurethane were obtained by a hydrothermal process under high-pressure conditions (1000 bar). In addition, three-dimensional structures based on nanostructured hybrid materials were obtained by the 3D Bioprinting method at room temperature, without heating the syringes through which the paste flowed and without heating the deposition substrate. In addition, the 3D deposition of HAp-PU hybrid powders was achieved using non-toxic water-soluble commercial polymers as binders and extrusion agents. Preformed nanostructured hydroxyapatite implants can be an alternative to existing implants for prosthetic orbital wall fractures, being cost-effective. Fabrication of customized implants is possible by using CT reconstruction images and $3 \mathrm{D}$ printing deposition of the implant.

Three-dimensional structures based on HAp-PU nanostructured hybrid powders obtained through the $3 \mathrm{D}$ Bioprinting method represent an absolute novelty in terms of material and process both nationally and at the European level. To our knowledge, there are no literature data or patents for the fabrication of orbital implants from hybrid powders of the hydroxyapatite-polymer type obtained in situ and then used in $3 \mathrm{D}$ printing deposition.

A market study showed that most commercial orbital implants use coralline HA, considered the gold standard for this type of implant. As an 
alternative, the present article proposed a new 3Dstructured material, namely the HAp-PU hybrid, which is easy for SMEs to implement.

\section{ACKNOWLEDGEMENTS}

This work was supported by UEFISCDI, ctr. 114/ 2014, Acronym ORBIMPLANT (2014-2017), and H2020-TWINN GA, No. 692216, Acronym SUPERMAT (2016-2018).

\section{OPEN ACCESS}

This article is distributed under the terms of the Creative Commons Attribution 4.0 International License (http://creativecommons.org/licenses/by/4.0/), which permits unrestricted use, distribution, and reproduction in any medium, provided you give appropriate credit to the original author(s) and the source, provide a link to the Creative Commons license, and indicate if changes were made.

\section{REFERENCES}

1. J. Gu, M. Meng, A. Cook, and M.G. Faulkner, Rob Auton Syst 32, 153 (2000).

2. L. Dubois, S.A. Steenen, P.J. Gooris, R.R. Bos, and A.G. Becking, Int. J. Oral Surg. 45, 41 (2016).

3. F. Baino and I. Potestio, Mater. Sci. Eng. C 69, 1410 (2016).

4. M.C. Goiato, M.F. Haddad, D.M. dos Santos, A.A. Pesqueira, P. do Prado Ribeiro, and A. Moreno, J. Craniofac. Surg. 21, 870 (2010)

5. Reconstructive procedures. Prosthetic Eye Implants: "A remarkable similarity was noticed between the porous structure of southern ocean coral species and that of human bone", https://www.drchipcole.com/prosthetic-eye-implants/.
Accessed 20 Sept 2018.

6. A.C. Perry, Ophthalmol. Clin. N. Am. 4, 173 (1991).

7. J.J. Dutton, Ophtalmology 98, 370 (1991).

8. J.P. Spirnak, N. Nieves, D.A. Hollsten, W.C. White, and T.A. Betz, Am. J. Ophthalmol. 119, 431 (1995).

9. C.K. You, S.H. Oh, J.W. Kim, T.H. Choi, S.Y. Lee, and S.Y. Kim, Key Eng. Mater. 240-242, 563 (2003).

10. H.N. Chia and B.M. Wu, J. Biol. Eng. 9, 4 (2015).

11. M. Prakasam, M. Popescu, R. Piticescu, and A. Largeteau, Fabrication Methodologies of Biomimetic and Bioactive Scaffolds for Tissue Engineering Applications.Scaffolding in Bioengineering - Materials, Technologies, Clinical Applications, ed. F. Baino (Rijeka: InTech, 2017), p. 3.

12. L.M. Popescu, R.M. Piticescu, A.M. Motoc, L.M. Voinea, S.L. Gradinaru (Istrate), D. Ulieru, and A. Topor, European Patent Request EP17020409.3-1109/336319 (2017), European Patent Bulletin number 2018/35.

13. M.A. Deriu, M.L. Popescu, M.F. Ottaviani, A. Danani, and R.M. Piticescu, J. Mater. Sci. 51, 1996 (2016)

14. L.M. Popescu, R.M. Piticescu, M. Petriceanu, M.F. Ottaviani, M. Cangiotti, E. Vasile, M.M. Dîrtu, M. Wolff, Y. Garcia, G. Schinteie, and V. Kuncser, Mater. Chem. Phys. 161, 84 (2015).

15. L.M. Popescu, R.M. Piticescu, A. Antonelli, C.F. Rusti, C.E. Carboni, C. Sfara, M. Magnani, V. Badilita, E. Vasile, R. Trusca, and T. Buruiana, J. Mater. Sci. Mater. Med. 24, 2491 (2013).

16. L.M. Popescu, C.F. Rusti, R.M. Piticescu, T. Buruiana, T. Valero, and S. Kintzios, J. Compos. Mater. 47, 603 (2013).

17. C.R. Tubío, F. Guitián, and A. Gil, J. Eur. Ceram. Soc. 36, 3409 (2016)

18. R. Khorramirouz, J.L. Go, C. Noble, S. Jana, E. Maxson, A. Lerman, and M.D. Young, Acta Histochem. 120, 282 (2018)

19. J. Li, Q. Xu, B. Teng, C. Yu, J. Li, L. Song, Y.X. Lai, J. Zhang, W. Zheng, and P.G. Ren, Acta Biomater. 42, 389 (2016). 